BULL. AUSTRAL. MATH. SOC.

$20-04,20 D 08,20 E 28$

VOL. $22(1980), 467-468$.

\title{
COMPUTATIONAL APPROACHES TO CERTAIN PROBLEMS \\ IN THE THEORY OF FINITE GROUPS
}

\author{
GREGORY BUTLER
}

In 1967 C.C. Sims announced a compact data structure which allows efficient computation in permutation groups of large degree and very large order. He developed algorithms which generate the data structure, and later developed very efficient backtrack searches for computing the centralizer of an element and the intersection of two subgroups. The techniques have also been used in the construction of several sporadic simple groups.

The thesis began as an investigation of these algorithms, and with the question whether they would be applicable to finite matrix groups. Several of the algorithms for permutation groups are refined. The data structure is generalized to encompass matrix groups, and analogues of Sims' algorithms are developed for matrix groups. In addition, further backtrack searches are developed notably to compute the normalizer of a subgroup, and Sylow subgroups of a group.

The theoretical description and proof of the algorithms is so formulated that the cases of permutation and matrix groups are treated simultaneously. The common skeleton of the backtrack searches is distilled out, thus isolating the main problem areas in the development of a backtrack search and leading to simple descriptions of the algorithms.

Other problems in computational group theory which are discussed are determining the conjugacy classes of elements of a group, enumerating the cosets of a subgroup, computing the elementary abelian subgroups of a

Received 22 August 1980. Thesis submitted to the University of Sydney, August 1979. Degree approved, June 1980. Supervisor: Dr J.J. Cannon. 
group, and computing the image and kernel of various "natural" homomorphisms.

The computational techniques developed were helpful in the determination of the maximal subgroups of the sporadic simple group of Held, which has order 4030387200 and minimal permutation representation of degree 2058 . This result forms the last chapter of the thesis. 